\title{
RELEVANCE FEEDBACK IN CBIR
}

Hongjiang Zhang, Zhong Su

Microsoft Research Asia

\begin{abstract}
A new focus in content-based image retrieval (CBIR) research is applying relevance feedback originally developed for text document retrieval, to improve the retrieval performance. This effort tries to bridge the gap between low-level image features and high-level semantic contents of images as this gap is the bottleneck of CBIR. We consider relevance feedback in CBIR a small sample-learning process in sparse image feature space. Almost all of the previously proposed methods fall well into such framework. In this paper, we present a brief overview of the current stateof-the-art of this topic, and present a framework of integrated relevance feedback and semantic learning in CBIR.
\end{abstract}

Key words: Relevance Feedback, Content-based image retrieval (CBIR), Machine Learning

\section{INTRODUCTION}

Having been actively researched on in the last decade [1][13][21], content-based image retrieval (CBIR) attempts to automate the process of indexing in image database. CBIR approaches work with descriptions based on inherent properties of images, such as colour, texture and shape. However, despite all the research efforts, the retrieval accuracy of today's CBIR algorithms is still very limited. Apart from many other difficulties, the bottleneck is the gap between low-level image features and semantic image contents. This problem stems from the fact that visual similarity measures, such as color histograms, in general do not necessarily match semantics of images human subjectivity. To make the problem even worse, people often have different semantic interpretations of the same image. 
Even the same person may have different perception about the same image at different times. To address this bottleneck, interactive relevance feedback techniques have been proposed. The key idea is that we should incorporate human perception subjectivity into the retrieval process and provide users opportunities to evaluate retrieval results and automatically refine queries on the basis of those evaluations. In the last few years, this research topic has become the focus in CBIR research community.

Relevance feedback, originally developed for textual document retrieval [18], is a supervised active learning technique used to improve the effectiveness of information systems. The main idea is to use positive and negative examples from the user to improve system performance. For a given query, the system first retrieves a list of ranked images according to a predefined similarity metrics. Then, the user marks the retrieved images as relevant (positive examples) to the query or not (negative examples). The system will refine the query based on the feedback and retrieves a new list of images and presents to user. Hence, the key issue in relevance feedback is how to incorporate positive and negative examples to refine the query and/or to adjust the similarity measure.

It is very interesting to notice that relevance feedback techniques in document retrieval were studied rather limitedly, it becomes the most active research topic in the last three years after being introduced into CBIR community. The chief fact contributed to this phenomenon is that the retrieval accuracy of general CBIR algorithm is so low that directly apply the relevance feedback framework developed for textual document retrieval is able to improve the accuracy significantly [3][20][26][27][28].

In this paper, we first survey the current state-of-the-art of CBIR relevance feedback research in terms of their natures and limitations. We then present a framework of integrated relevance feedback that support active learning, automated annotation with semantic propagation and cross-modality query expansion. In this framework, while the user is interacting with the system by providing feedbacks in a query session, a progressive learning process is activated to propagate the keyword annotation from the labelled images to un-labelled images as the system refines the retrieval. In addition, a cross-modality query expansion scheme is implemented to improve the retrieval performances significantly either a query is initiated with a keyword or an example image.

\section{RELEVANCE FEEDBACK IN CBIR-A REVIEW}

There are many issues in relevance feedback approaches to CBIR, such as learning schemes, feature selection, index structure and scalability. 
Instead of giving an exhaustive survey of each published relevance feedback algorithms for CBIR in term of their advantages and limitations, we focus our discussions with the consideration that relevance feedback in CBIR is a machine learning problem and extend our survey in detail in respect to learning and search natures of each algorithm. To give some background, we first provide an overview of classic relevance feedback approaches to document retrieval.

\subsection{Classic Relevance Feedback Algorithms}

The early relevant feedback schemes for ICBR were mainly adopted from those developed for classical textual document retrieval. These approaches can be classified into two approaches: query point movement (query refinement) and re-weighting (similarity measure refinement) [1]. Both of them were developed based on vector model, the most popular models used in information retrieval [22].

The query point movement method essentially tries to improve the estimate of the "ideal query point" by moving it towards good examples point and away from bad example points. The frequently used technique to iteratively improve this estimation is the Rocchio's formula given below for sets of relevant documents $D^{\prime}{ }_{R}$ and non-relevant documents $D^{\prime}{ }_{N}$ given by the user [18].

$$
Q^{\prime}=\alpha Q+\beta\left(\frac{1}{N_{R^{\prime}}} \sum_{i \in D_{R}^{\prime}} D_{i}\right)-\gamma\left(\frac{1}{N_{N^{\prime}}} \sum_{i \in D_{N}^{\prime}} D_{i}\right)
$$

where $\alpha, \beta$, and $\gamma$ are suitable constants; $N_{R^{\prime}}$ and $N_{N}$, are the number of documents in $D^{\prime}{ }_{R}$ and $D^{\prime}{ }_{N}$ respectively. This technique is implemented in the MARS system [19]. Experiments show that retrieval performance can be improved considerably by using such relevance feedback approaches.

The basic idea behind the re-weighting method is to enhance the importance of the dimensions of a feature that help in retrieving the relevant images and reduce the importance of those dimensions that hinder this process. The MARS system implemented a slight refinement to the re-weighting method called the standard deviation method [19].

Recently, more computationally robust methods that perform global feature optimization have been proposed. The MindReader retrieval system designed by Ishikawa et al. [10] formulates a minimization problem on the parameter estimating process. Unlike traditional retrieval systems whose distance function can be represented by ellipses aligned with the coordinate axis, the MindReader system proposed a distance function that is not necessarily aligned with the coordinate axis. Therefore, it allows for correlations between attributes in addition to different weights 
on each component. A further improvement over this approach is given in [20]. In their CBIR system, it not only formulates the optimization problem but also takes into account the multi-level image model.

\subsection{Visual Features vs. Semantics}

All the approaches described in Section 2.1 perform relevance feedback at the low-level feature vector level by basically replacing keywords with features when adopting the vector model developed for document retrieval. While these approaches do improve the performance of ICBR, there are severe limitations. The inherent problem is that the low-level features are often not as powerful in representing complete semantic content of images as keywords in representing text documents. Furthermore, users often pay more attention to the semantic content (or a certain object/region) of an image than to the background and other, the feedback images may be similar only partially in semantic content, but may vary largely in low-level features. Hence, using low-level features alone may not be effective in representing users' feedbacks and in describing their intentions.

There have been efforts on incorporating semantics in relevance feedback for image retrieval. The framework proposed in [13] attempted to embed semantic information into a low-level feature based image retrieval process using a correlation matrix. In this effective framework, semantic relevance between image clusters is learnt from user's feedback and used to improve the retrieval performance. In other word, the framework maintains the strengths of feature-based image retrieval while incorporating learning and annotation in the relevance feedback processes.

The FourEye system by Minka and Picard[19] and the PicHunter system by Cox, et al [3] made use of hidden annotation through learning process. However, they excluded the possibility of benefiting from good annotations, which may lead to a very slow convergence.

As to be presented in Section 3 and 4, our proposed framework integrates both semantics and low-level features into the relevance feedback process in a new way. Only when the semantic information is not available, our method is reduced to one of the previously described low-level feedback approaches as a special case.

\subsection{With vs. Without Memory}

Another disadvantage in the classic relevance feedback approaches, such as [19], [20], is that the captured knowledge in the relevance feedback processes in one query session is not memorized to continuously improve the retrieval. That is, even with a same query, a user will have to 
go through the same, often tedious, feedback process to obtain the same result, despite the fact the user has given the same query and feedbacks before. Strictly speaking, there is no learning or only limited learning in such systems as there is no knowledge accumulation across different query sessions. To overcome these limitations, another school of ideas is to using learning approaches to memorize users' subjectivities in relevance feedback process. The challenge in this approach is how to memorize knowledge learned and how to handle the inconsistency of content subjectivities across difference users and/or across different query sessions of the same user.

The framework proposed in [13] was the attempt to explicitly memorize learned semantic information to improve CBIR performance. In this effective framework, users' subjectivities in the form of semantic relevance between image clusters is learnt from user's feedback and memorized in a correlation matrix. Experiments have shown that this new framework effective utilizes the knowledge learnt from previous queries to reduce the number of iterations in following queries. Annotation-based approaches, such as [14], are ones with explicit.

\subsection{Learning vs. Classification}

Relevance can be considered as a leaning problem- a user provides feedback examples from the retrieval result of a query and system learns from such examples to refine retrieval results. According to Mitchell's [31] definition, machine learning is concerned with the question of how to construct computer programs that automatically improve with experience. In this view, any task that could be improved with respect to certain performance measure based on some experience can be considered the machine-learning task. In CBIR, relevance feedback is a task to improve the retrieval performance and the experience here is user's providing feedback examples. Almost all of the previous state-of-the-art relevance feedback methods for CBIR are classic machine-learning methods, such as decision tree learning [32], artificial neural networks [33], Bayesian learning [3][28], kernel based learning [30] and etc. However, as users are usually reluctant to provide a large number of feedback examples, the number of training samples is very small, typically less than ten in each round of feedback session. On the contrary, feature dimensions in CBIR systems are usually high. Hence, the crucial issue in performing relevance feedback in CBIR systems is how to learn from small training samples in $a$ very high dimension feature space. This fact makes many learning methods, such as decision tree learning and artificial neural networks, not suitable for CBIR. 
The original query-movement method represented by the Rocchio's formula and re-weighting method [19][21] are both simple learning methods. Compared with other learning methods, Bayesian learning shows its advantages in CBIR task. Many works have been done in this field [3][26][28] and achieved remarkable performances. Naïve Bayesian [15]; Optimized Bayesian classification [28]; Bayesian inference network and Monte-Carlo methods [3], almost all aspects of Bayesian learning have been touched in researching for effective relevance feedback schemes for CBIR. Vasconcelos and Lippman [28] treated feature distribution as a Gaussian mixture and used Bayesian inference for learning during feedback iterations in a query session. Under their feature distribution model, regional matching can be supported. The potential problems of their methods are computing efficiency and complex data model that leads to too many parameters need to be estimated with very limited samples. Cox et al. [3] used Bayesian framework to do target search by attempting to minimize number of feedback iterations by entropy estimation.

Some researchers consider relevance feedback process in CBIR as a pattern recognition or classification problem. Under such consideration, the positive and negative examples provided by user can be treated as training examples and a classifier could be trained. Then, such classifier can separate all data set into relevant and irrelevant groups. It seemed that many existing pattern recognition tools could be adopted for this task and many kinds of classifiers have been experimented, such as linear classifier [34], nearest-neighbour classifier [35], Bayesian classifier [27], support vector machines(SVM) [30], and so on. In this category, the most popular algorithm is represented by [30] where SVM classifier is trained to divide the positive and negative examples. Then such SVM classifier will classify all images in database into two groups: relevant and irrelevant groups to a given query.

Here we will not enumerate pervious works. Rather, we focus on the discussion whether or not it is reasonable to treat relevance feedback process in CBIR as a pattern recognition problem. In a typical pattern recognition case, there usually is a clear class structure. In other words, each item in a data set belongs to one or a number of clearly defined classes. The classification algorithm usually has very clear goal: to separate different classes as much as possible in whole data set. However, the case of content based image retrieval is different from such typical cases. In most cases, there is no pre-defined class structure. Even if there is a pre-defined class structure, it is still often difficult to determine an image item belong to which category. This is due to human subjectivities and inconsistence in human perception. Hence, the classification often 
lacks no clear goal. From application point of view, such classificationbased methods may improve the retrieval performance in some constrained contexts; but they will be limited when applied to general purpose image databases.

\subsection{Category Search vs. Target Search}

Actually, this topic is not directly related with feedback. The issue here is what a user needs when using a CBIR system. User may look for similar images or a particular image. Before Cox et al. [3] presented the idea of target search, category search was the only search style in CBIR system. In category search, user provides the sample image and wants to find those images similar in contents with the sample. Under relevance feedback framework, user can make the relevance judgement to iteratively refine the retrieval performance. User can provide binary judgement of positive and negative examples [27][28]. Single judgement also been widely used where only positive examples are considered [10][26]. Some systems provide more complex interactive methods where relevance and irrelevance degree are to be considered [20]. Different level represents different degree of how relevant or irrelevant of the image comparing with the query image.

Target search [3] is another kind of scenario. Under such scenario, a user looks for a particular target image and the positive examples he provides in one retrieval session are those closer to the target image he wants. The work by Cox, et al, was the first one presented such idea, in which Bayesian inference was applied to answer the question. However, a number of practical problems need to be considered under such search style. First, which image is closer to the target is a challenging question for user to answer. Second, similar in image contents may be far away from each other in a feature space. Hence, it may be mission impossible for relevance feedback to solve the target search problem.

\section{OUR PROPOSED FRAMEWORK}

As discussed in the last section, the most crucial issues in relevance feedback of CBIR systems are the following. 1) How to learn effectively from small sets of feedback samples; 2) How to accumulate knowledge learned from feedback; 3) How to integrate low-level visual and high-level semantic features in query and feedbacks. An effective relevance feedback system should provide effective solutions to address these three issues, in addition many others. 
In addition, there typically are two different modes of user interactions involved in image retrieval systems. In one case, the user types in a list of keywords representing the semantic contents of the desired images. In the other case, the user provides a set of examples images as the input and the retrieval system will try to retrieve other similar images. In most image retrieval systems, these two modes of interaction are mutually exclusive. We argue that combining these two approaches and allow them to benefit from each other yields a great deal of advantage in terms of both retrieval accuracy and ease of use of the system.

To address all of above-mentioned four issues, we have proposed a CBIR framework with integrated relevance feedback and query expansion, in which the semantic-based index and relevance feedback are seamlessly integrated with those based on low-level feature vectors. Figure 1 illustrates the proposed framework. As illustrated, the proposed relevance feedback framework consists of a semantic network which links images to semantic annotations in a database, a similarity measure that integrating both semantic features and image features, and a machine learning algorithm to iteratively update the semantic network and to improve the system's performance over time.

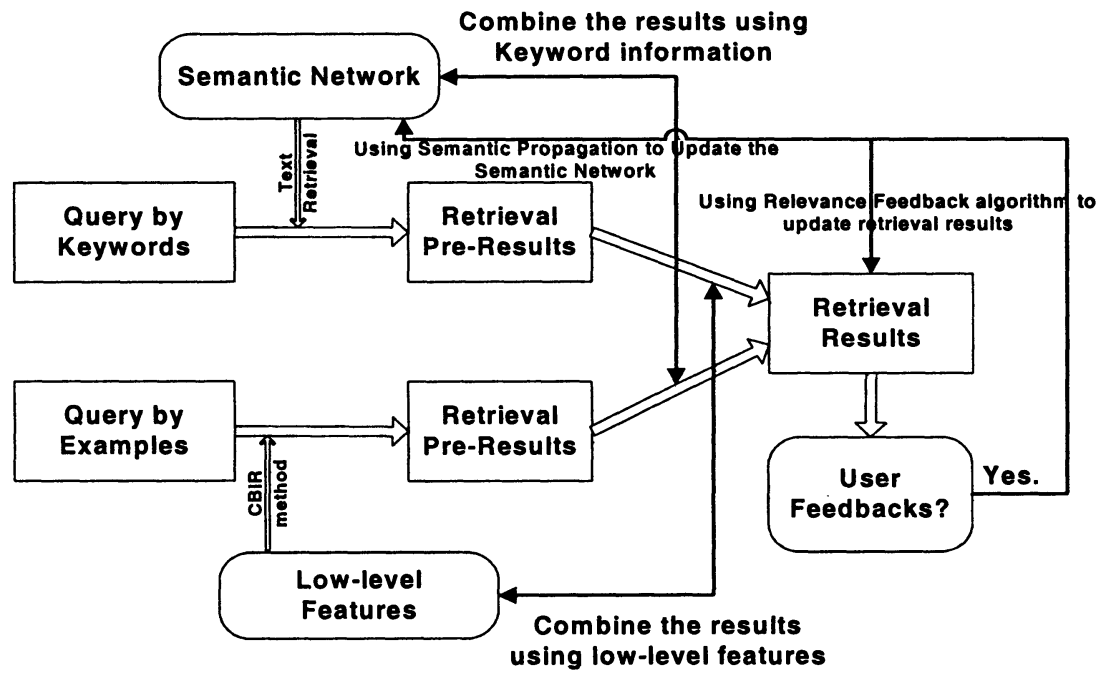

Figure 1. The Proposed Framework of Integrated Relevance Feedback and Query Expansion.

The framework supports both query by keyword and query by image example through semantic network and low-level feature indexing. Crossmodality query expansion is supported. That is, the retrieved images based on keyword search are considered as the positive examples, based on which the query is expanded by features of these images. In this way, 
the system extends a keyword-based query into feature-based queries to expand the search range. For query by image example, similar procedure takes effect to extend the retrieval from feature space to semantic space. In this way, user input information is utilized as much as possible to improve the retrieval performance. More importantly, there is a progressive learning process to propagate the keyword annotations from the labelled images to un-labelled ones during the feedback. In this way, more and more images are implicitly labelled by keywords by the semantic propagation process. In addition to supporting keyword search, this annotation propagation process also the retrieval system accumulated users' feedback information so that it will be improve performance of future retrieval requests.

The proposed framework has an integrated relevance feedback scheme in which both low-level feature based and high-level semantic feedback are performed. We define a unified metric function $G$ to measure the relevance between query $Q^{\prime}$ and any image $j$ within an image database in terms of both semantic and low-level feature content, where $Q^{\prime}$ includes the original query and users' feedback information.

$$
G\left(j, Q^{\prime}\right)=\alpha \cdot \operatorname{sim}_{k}\left(j, Q^{\prime}\right)+(1-\alpha) \cdot \operatorname{sim}_{f}\left(j, Q^{\prime}\right)
$$

where $\alpha \in[0,1]$ is the weight of the semantic relevance in the overall similarity measurement which can be specified by users. The larger $\alpha$ is, the more important the semantic relevance will play in the overall similarity measurement. $\operatorname{sim}_{f}\left(j, Q^{\prime}\right)$ and $\operatorname{sim}_{k}\left(j, Q^{\prime}\right)$ are the low-level feature similarity and semantic similarity between image $j$ and $Q^{\prime}$, respectively.

The semantic network is a two-layered structure. The top layer is represented by a set of keywords having links to the images in the database. Weights are assigned to each individual link, as shown pictorially in

Figure 2. The degree of relevance of the keywords to the associated images' semantic content is represented as the weight on each link. This layer is what we need in keyword relevance feedback and will be updated during the semantic propagation. Bottom layer is a keyword thesaurus to construct the connection between different keywords.

Semantic based relevance feedback is performed in keyword vector space. Each dimension is represented by a specific keyword. For each image, there are corresponding text descriptors composing by a text feature vector. The vector can be obtained by manual labelling or assigned according to its URL, filename, ALT text, hyperlinks and surrounding text if the image is downloaded from website, more keywords 
can be assigned. Of course, if no keyword information to the image, the corresponding feature vector is set to null. The semantic propagation method is used to annotate such image during user's feedback iteration, which will be described in later session.

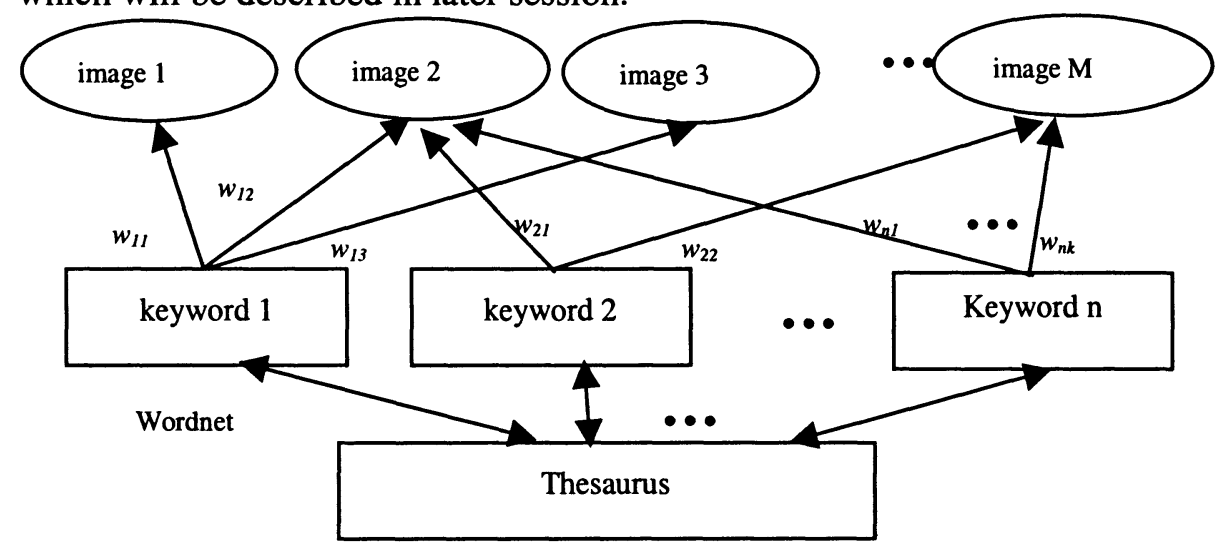

Figure 2. Semantic Network.

\section{PROBABLISTIC PROPAGATION SCHEME}

As illustrated in Figure 2, the more images are annotated (correctly), the better the system retrieval performance will be. However, the reality is human labelling of images is tedious and expensive, hence not a feasible solution, which was what motivated CBIR research fifteen years ago. To address this issue, a probabilistic progressive keyword propagation scheme is proposed in our framework to automatically annotate images in the databases in the relevance feedback process utilizing based a small percentage of annotated images.

We assume that initially only a few images in a database have been manual labelled with keywords and the retrieval is performed mainly based on low-level features. As stated before, the initial keywords annotation can be from web through the crawler when the images are from the Web, or labelled by humans. While the user is interacting with the system by providing feedbacks in a query session, a progressive learning process is activated to propagate the keyword annotation from the labelled images to un-labelled images so that more and more images are implicitly labelled by keywords. In this way, the semantic network is updated in which the keywords with a majority of user consensus will emerge as the dominant representation of the semantic content of their associated images. As more queries are inputted into the system, the system is able to expand its vocabulary. Also, through the propagation process, the keywords that 
represent the actual semantic content of each image will receive a large weight.

There are two major issues in keyword propagation: which images and which keyword(s) should be propagated during a query session.

To answer the first question, a probability model, based on Bayesian learning, is proposed. We assume that, (1) all positive examples in one retrieval session belong to the same semantic class with common semantic object(s) or meaning(s); and (2) the features from the same semantic class follows the Gaussian or Mixture Gaussian distributions. Therefore, all positive feedbacks in a query session are used to calculate and update the parameters of the corresponding semantic Gaussian classes. Then, the probability of each image in the database belonging to such semantic class is calculated. The common keywords in positive examples are propagated to the images with very high probability belonging to this class.

As we can see, the propagation framework uses the same procedure as the feedback algorithm in low-level features. The only difference is that for low-level feature feedbacks, the calculated probability is used for the ranking of an image in retrieval candidate list, while here it is used to determine if an image should be in the propagation candidate list.

Same as the feedback algorithm in low-level feature space, $p\left(c_{i}\right)$, can be calculated [26]. Then the propagation candidate set $S$ is obtained as follows:

$$
S=\left\{c_{1}, \ldots, c_{k}\right\}, \text { where } p\left(c_{i}\right)>\psi
$$

$\psi$ is a constant threshold which can be estimated by the training process. The weight associates with the propagated keyword $i$ and the image $m$ $w_{i m}=p\left(c_{m}\right)$. More complex distribution model, for example Mixture Gaussian, may be used in this propagation framework. However, because the user's feedback examples in practice are often very few, complex models will leads into much more parameter estimation errors as there are more parameters to be estimated.

Also, to determine which keyword(s) should be propagated when an image is associated with multiple keywords, a region-based approach is evolved. Unlabelled images to be propagated are firstly segmented into regions. By analysing the feature distribution of the segmented regions, a probability association between each segmented regions and annotated keywords is set up for labelled images by region-based relevance feedback approach. Then, each keyword of labelled image was assigned to one or several regions of the image with certain probabilities. The region-based feedback framework we used is described in [11]. It is also a probabilistic feedback approach. Currently, all common keywords will be propagated to the candidate list. 


\section{EXPERIMENTAL RESULTS}

The image set we used is the Corel Image Gallery. 10,000 images of 79 semantic categories are selected to calculate the performance statistics. 200 random selected images compose the test query set. Whether a retrieved image is correct or incorrect is judged according to the ground truth. Three types of colour features and three types of texture features are used in our system. Feedback process is running as follows: Given a query example from the test set, a different test image of the same category as the query is used in each round of feedback iteration as the positive example for updating the Gaussian parameters. For negative feedback process, the first two irrelevant images are assigned as negative examples. The accuracy is defined as:

$$
\text { Accuracy }=\frac{\text { relevant images retrieved in top } N \text { returns }}{N}
$$

Several experiments have been performed as follows. First, our Bayesian feedback scheme is compared with previous feedback approaches presented by Nuno [28] and Rui [20][21]. This comparison is done in the same feature space. Error! Reference source not found. shows that the accuracy of our Bayesian feedback method becomes higher than the other two methods after two feedback iterations. This demonstrates that the incorporated Bayesian estimation with the Gaussian parameter-updating scheme is able to improve retrieval effectively.

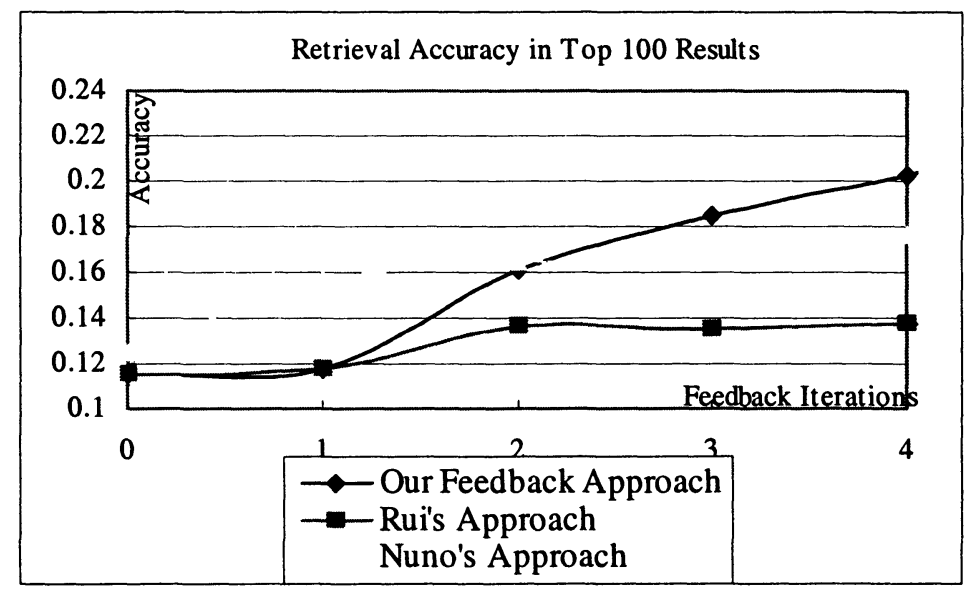

Figure 3: Retrieval accuracy for top 100 results in original feature space. 
To demonstrate the performance of the semantic propagation, we design the following experiment. We have annotated the 200 images in the query set by its category names. So, only one keyword is associated to one query image and other images in database have no keyword annotations. During the test, each query image was used twice. Then we compared the retrieval performance as shown in Figure 4. It is seen that for feedback with propagation, the retrieval accuracy is much higher than the original one without it. This is because, without propagation, the system has no 'memory'. Every time when user re-submits the same query, user has to start from the very low accuracy. However, when a system has propagation ability, latter user can utilize the accumulated knowledge from previous feedback iterations. In other words, system has the leaning ability and will be smarter with more users' interactions.

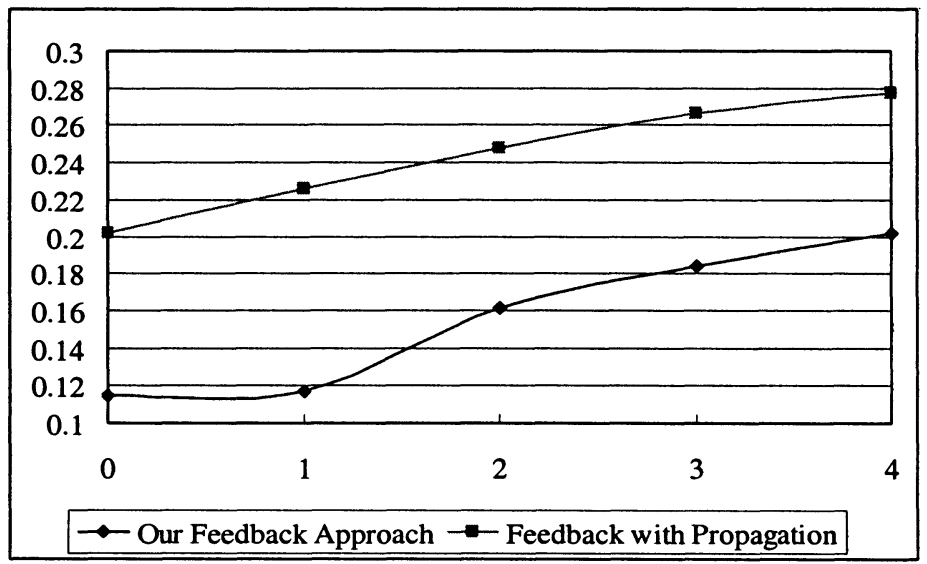

Figure 4: Retrieval accuracy for top 100 results performance between feedback without propagation and feedback with propagation scheme.

\section{CONCLUSION}

In this paper, we present the framework design of our image retrieval system which is build based on relevance feedback and probabilistic keyword propagation. Our method utilizes both semantic and low-level feature properties of every feedback image in refine the retrieval, while in the meantime, learning semantic annotations of each image. While the user is interacting with the system by providing feedbacks in a query session, a progressive learning process is activated to propagate the keyword annotation from the labelled images to un-labelled images so that more and more images are implicitly labelled by keywords at certain 
probabilities. In this way, more and more images are implicitly labelled by keywords by the semantic propagation process. Thus, such process will improve the retrieval performance in future, either query by image examples or by keywords. This new framework makes the image retrieval system to be superior over either the classical CBIR or text-based systems.

\section{REFERENCES}

[1] Buckley, C., and Salton, G. "Optimization of Relevance Feedback Weights," in Proc of SIGIR' 95 .

[2] Chandrasekaran, S. et al. "An Eigenspace Update Algorithm for Image Analysis". CVGIP: Graphical models and image processing journal, 1997.

[3] Cox, I. J., Minka, T. P., Papathomas, T. V. and Yianilos, P. N. "The Bayesian Image Retrieval System, PicHunter: Theory, Implementation, and Psychophysical Experiments" IEEE Transactions on Image Processing -- special issue on digital libraries, 2000.

[4] Deng, Y., Manjunath, B. S. and Shin, H., "Color Image Segmentation", in Proc. IEEE Computer Society Conference on Computer Vision and Pattern Recognition, CVPR '99, Fort Collins, CO, vol.2, pp.446-51, June 1999.

[5] Diamantaras, I., and Kung, S.Y. "Principal Component Neural Networks, Theory and Applications" John Wiley \& Sons. Inc. 1996

[6] Duda, R. O., and Hart, P. E. (1973). "Pattern Classification and Scene analysis." New York: John Wiley \& Sons.

[7] Faloutsos, C. and Lin, K., "Fastmap: A Fast Algorithm for Indexing, Data-mining and Visualization of Traditional and Multimedia "In Proc. of SIGMOD, pages 163174, 1995.

[8] Flickner, M. et al. "Query by Image and Video Content: The QBIC system." Computer. Vol 28, pp23-32, 1995

[9] Fukunaga, K., "Introduction to Statistical Pattern Recognition" 2nd Edition, Academic Press 1990

[10] Ishikawa, Y., Subramanya R., and Faloutsos, C., "Mindreader: Query Databases Through Multiple Examples," In Proc. of the 24th VLDB Conference, (New York), 1998.

[11] Jing, F., Zhang, B., Lin, F., Ma, W. and Zhang, H. "A Novel Region-Based Image Retrieval Method Using Relevance Feedback", 3rd Intl Workshop on Multimedia Information Retrieval (MIR 2001), Ottawa, Canada, October 5, 2001.

[12] Kirby, M. and Sirovich, L. "Application of the Karhunen-Loeve procedure for the characterization of human faces", In IEEE Transactions on Pattern Analysis and Machine Intelligence, vol. 12, no. 1, pp. 103-108, January 1990.

[13] Lee, C., Ma, W. Y., and Zhang, H. J. "Information Embedding Based on user's relevance Feedback for Image Retrieval," Technical Report HP Labs, 1998.

[14] Lu, Y., Hu, C., Zhu, X., Zhang, H. and Yang, Q. "A Unified Framework for Semantics and Feature Based Relevance Feedback in Image Retrieval Systems", The 8th ACM Multimedia International Conference, November 2000, Los Angeles, CA.

[15] Meilhac, C. and Nastar, C. "Relevance Feedback and Category Search in Image Databases", IEEE International Conference on Multimedia Computing and Systems, Italy, 1999. 
[16] Ng, R. and Sedighian, A.. "Evaluating Multi-dimensional Indexing Structures for Images Transformed by Principal Component Analysis". Proc. SPIE Storage and Retrieval for Image and Video Databases, 1996.

[17] Brunelli, R. and Mich, O. "Image Retrieval by Examples". IEEE Trans. On Multimedia. Vol. 2. No. 3, September 2000.

[18] Rocchio, Jr., J. J.(1971). Relevance Feedback in Information Retrieval. In The SMART Retrieval System: Experiments in Automatic Document Processing (Salton, G. eds ) pp313-323. Prentice-Hall.

[19] Minka, T. and Picard, R. "Interactive Learning using a 'Society of Models'", Pattern Recognition, 30(4), 1997.

[20] Rui, Y., Huang, T. S., and Mehrotra, S. "Content-Based Image Retrieval with Relevance Feedback in MARS," in Proc. IEEE Int. Conf. on Image proc., 1997.

[21] Rui, Y., and Huang, T. S. "A Novel Relevance Feedback Technique in Image Retrieval," ACM Multimedia, 1999.

[22] Salton, G., and McGill, M. J. "Introduction to Modern Information Retrieval," McGraw-Hill Book Company, 1983.

[23] Shaw, W. M. "Term-Relevance Computation and Perfect Retrieval Performance," Information processing and Management.

[24] Sheikholeslami, G., Chang, W. and Zhang, A. "Semantic Clustering and Querying on Heterogeneous Features for Visual Data." 6th ACM International Multimedia Conference, Bristol, UK, September 1998.

[25] Stone, H. S. and Li, C. S. "Image Matching by Means of Intensity and Texture matching in the Fourier domain," in Proc. IEEE Int. Conf. Image Processing. Santa Barbara. CA. Oct 1997.

[26] Su, Z., Li, S., Zhang, H. "Extraction of Feature Subspaces for Content-Based Retrieval Using Relevance Feedback" ACM Multimedia 2001, Ottawa Canada

[27] Su, Z., Zhang, $\mathrm{H}$ and Ma, S. "Relevant Feedback using a Bayesian Classifier in Content-Based Image Retrieval” SPIE Electronic Imaging 2001, January 2001, San Jose, CA

[28] Vasconcelos, N., and Lippman, A. "Learning from User Feedback in Image Retrieval Systems" NIPS'99, Denver, Colorado, 1999.

[29] Zhu, L. and Zhang, A. "Supporting Multi-example Image Queries in Image Databases" IEEE International Conference on Multimedia and Expo, July, New York City, NY.

[30] Tong S. and Chang E. "Support Vector Machine Active Leaning for Image Retrieval" ACM Multimedia 2001, Ottawa, Canada.

[31] Mitchell. T. "Machine Learning". McCraw Hill, 1997

[32] MacArthur, S.D.; Brodley, C.E.; Shyu, C.-R. "Relevance feedback decision trees in content-based image retrieval" IEEE Workshop on Content-based Access of Image and Video Libraries, 2000. Page(s): $68-72$

[33] Laaksonen, J. Koskela, M. and Oja, E, "PicSOM: Self-Organizing Maps for Content-Based Image Retrieval", Proceedings of International Joint Conference on NN, July, 1999.

[34] Wu, Y. Tian, Q. and Huang, T.S, “Discriminant EM algorithm with Application to Image Retrieval”, IEEE CVPR, South Carolina, 2000.

[35] Wu, P. and Manjunath, B. S., “Adaptive Nearest Neighbour Search for Relevance Feedback in Large Image Database", ACM Multimedia conference, Ottawa, Canada, 2001. 